This is the Author's Accepted Manuscript version has been uploaded in accordance with the publisher's self-archiving policy. This is a paper accepted by Future Medicine Ltd. for publication in Pharmacogenomics.

For full bibliographic citation, please refer to the version available at www.futuremedicine.com.

Available first online on 1 May 2015 at http://dx.doi.org/10.2217/PGS.15.20. 


\title{
Pharmacometabonomics in Humans: A new Tool for Personalised Medicine
}

Jeremy R Everett

Medway Metabonomics Research Group,

University of Greenwich

Chatham Maritime

UK

ME4 4TB

telephone: $+44(0) 2083318323$

correspondence to Jeremy Everett at j.r.everett@greenwich.ac.uk

\begin{abstract}
Summary
Pharmacogenomics is now over 50 years old and has had some impact in clinical practice, through its use to select patient subgroups who will enjoy efficacy without side effects when treated with certain drugs. However, pharmacogenomics, has had less impact than initially predicted. One reason for this is that many diseases, and the way in which the patients respond to drug treatments, have both genetic and environmental elements. Pure genomics is almost blind to the environmental elements. A new methodology has emerged, termed pharmacometabonomics that is concerned with the prediction of drug effects through the analysis of predose, biofluid metabolite profiles, which reflect both genetic and environmental influences on human physiology. In this review we will cover what pharmacometabonomics is, how it works, what applications exist and what the future might hold in this exciting new area
\end{abstract}

Keywords: metabolomics • metabonomics - NMR spectroscopy $•$ personalized medicine $•$ pharmacometabolomics $\cdot$ pharmacometabonomics $\cdot$ precision medicine

\section{Introduction}

Drug therapy is challenged by the fact that many drugs do not work, or are unsafe, in significant segments of the patient population.[1] The lack of safety is particularly worrying. It has been estimated that adverse drug reactions (ADRs) in hospitalized patients are the fourth to sixth largest cause of death in the USA.[2] The cost alone to the US economy could be up to $\$ 30$ billion per annum, not to mention the very significant human suffering and loss.[3-6] Personalised medicine, also known as Precision Medicine or Stratified Medicine,[7] seeks to avoid these issues through the development of methodologies that enable clinicians to know in advance of dosing which drugs will be both efficacious and safe in their patients.

Pharmacogenomics is focused on the prediction of the effects of drugs on the body, and conversely, of the effects of the human body on the drug, through the use of genomic profiling. Pharmacogenomics is now over 50 years old and has started to have some impact in clinical practice, through its use to select patient subsets for whom efficacy with minimal side effects will be achieved when treated with certain drugs. A good example of this is the use of pharmacogenomics to help with dose-setting for the antithrombotic agent warfarin. Dose-setting is particularly important in this context, as an 
overdose of the drug can cause bleeding in the patient. Warfarin works via the inhibition of vitamin K epoxide reductase complex 1 (VKORC1) but is significantly metabolised in the body by human cytochrome P450s (CYPs), especially CYP2C9. Warfarin metabolism is reduced by 30 to $50 \%$ in carriers of the $C Y P 2 C{ }^{*} 2$ allele and by $90 \%$ in carriers of the $C Y P 2 C 9 * 3$ allele, so that in the absence of screening, a patient could easily be overdosed and suffer bleeding induced by warfarin. Genetic screening for single nucleotide polymorphisms in VKORC1 and CYP2C9 has been shown to lead to more appropriate dosing and fewer side effects. However, there is still a lack of clinical evidence from randomized control trials (RCTs) demonstrating this benefit with statistical significance and, even in this strong case, pharmacogenomics testing was not recommended for clinical practice.[8]

The lack of conclusive confirmation of the value of pharmacogenomics in RCTs is a common finding in recent reviews of pharmacogenomics trials across a number of therapeutic areas, including cardiovascular,[8] diabetes[9] and depression.[10] Whilst genetic variation has been associated with treatment response, there is little evidence to validate that these associations can be used in clinical practice to guide patient treatment choices. In addition, many trials had issues in their design, including lack of clear definition of outcome phenotypes (especially for ADRs),[11] being underpowered, having no control groups, lacking independent replication and failing to deal with false discovery rates adequately.[9] Thus, the promise of pharmacogenomics is clear, but it has yet to deliver in clinical practice.

It is now well known that many diseases have both genetic and environmental causes, and moreover, the interaction between drugs and the human body also involves both genomic and environmental elements. Pure human genomics is almost blind to these environmental elements and this is another reason that pharmacogenomics alone will be less sensitive or specific than desired.

In the past ten years a new methodology has emerged, termed pharmacometabonomics that is concerned with the prediction of drug effects through the use of pre-dose metabolic profiles of biofluids, as opposed to genomic profiles of tissues or biofluids. These biofluid metabolite profiles reflect the exact status of the human body at the point of sampling, as opposed to genomic information that indicates what might happen to a person's physiology, at some future time point, and with many caveats. Biofluid metabolite profiles reflect directly both genetic and environmental influences, especially from the microbiome, on human physiology. In this review we will cover what pharmacometabonomics is and how it works, what the current status of human pharmacometabonomics is, and what the future might hold in this exciting new area.

\section{Metabonomics}

Metabonomics is defined as 'The study of the metabolic response of organisms to disease, environmental change or genetic modification'.[12] Metabonomics was defined in 1999 by Jeremy Everett and Jeremy Nicholson in order to provide an holistic framework for a series of genomic, proteomic and metabolic profiling studies that were conducted between Pfizer and Imperial College with the aim of finding biomarkers of pre-clinical drug safety. The term thus covered the sort of biofluid analysis experiments that had been ongoing for some years using detection technologies such as nuclear magnetic resonance (NMR) spectroscopy and liquid chromatography - mass 
spectrometry (LC-MS).[13] Shortly afterwards, the alternative term metabolomics was defined as a 'comprehensive analysis in which all the metabolites of a biological system are identified and quantified'[14] Originally, the term metabolomics tended to be used for plant or cell profiling, or experiments conducted with MS detection, and metabonomics for studies on animal or human samples, or those experiments with NMR detection. However, these days, the terms are used interchangeably. Given the fractal complexity of biological fluids and the almost limitless number of metabolites to be observed, the definition of metabolomics is not practical. We will stick to the original term metabonomics and its definition throughout this review.

Metabonomics enjoys a great deal of success in the analysis of changes in the biofluids of animals and humans due to interventions such as drug treatment, diet change, exercise and the impact of disease.[15] However, it was frequently observed, by many groups and over a long time period that highly variable results could be obtained, especially in pre-clinical safety studies on rats. Understanding this phenomenon, instead of dismissing it as 'biological variability', gave rise to the discovery of pharmacometabonomics.

\section{Pharmacometabonomics}

In the late 1990s Pfizer and Imperial College conducted a series of pre-clinical experiments designed to detect safety signals from drugs earlier than was then possible, using a combination of metabonomics, proteomics and genomics, as well as conventional biochemical assays. The variability of post-dose, biofluid metabolite profiles in some of the metabonomics experiments involving drug dosing in rats could be so extreme that it would appear that one sub-group of animals had been dosed and another had not. In the course of a Pfizer - Imperial College collaboration review meeting in Amboise, France on October $18^{\text {th }} 2000$, the issue of variable post-dose, endogenous and xenobiotic, metabolite profiles had emerged as a problem again. The proposal was made at the meeting that the post-dose variability was related to pre-dose differences between the animals. This was a novel and radical proposal at the time: the animals used in the studies were all of the same strain, the same weight, the same age, and treated identically. Nevertheless, the concept was followed up and experiments were designed to test the hypothesis that there was a direct association between the metabolic status of the animals pre-dose, and the variation observed in post-dose responses. These experiments culminated in a study of variability of drug metabolism and of toxicity in Sprague Dawley rats dosed with the common analgesic, paracetamol. The hypothesis was proven, pharmacometabonomics was discovered, and the results were published in Nature in 2006.[16]

On the basis of this work, pharmacometabonomics was defined as 'the prediction of the outcome (for example, efficacy or toxicity) of a drug or xenobiotic intervention in an individual based on a mathematical model of pre-intervention metabolite signatures'[16] Pharmacometabonomics is thus the metabolic profiling equivalent of pharmacogenomics. The concept of pharmacometabonomics is that some animals or humans will respond differently to drug administration because their differentiated pre-dose phenotypes predisposes them to do so. Pre-dose phenotypes are influenced by the subjects' genome and by their environment, and in particular by the status of their microbiome and its interaction with their genome. Similarly to the terminology for metabonomics, there has been a later adoption of the term pharmacometabolomics. In 
general, these are used interchangeably; we will use the original terminology and definition throughout this review.

\section{Conducting Pharmacometabonomics Experiments}

The aim of a pharmacometabonomics experiment is to establish correlations between variations in the pre-dose biofluid metabolite profiles of the subjects and their postdrug dose responses. This is done by careful measurement of the metabolite profiles of biofluids such as urine or blood plasma both before, and at intervals after, the drug administration. The measurement of the metabolite profiles can be done in a targeted or untargeted fashion. In a targeted experiment, a limited number of metabolites of defined chemical type are measured and their levels are quantified. This is typically done with technologies such as UPLC-MS with a triple quadrupole MS, by liquid chromatography - electrochemical analysis (LC-ECA), which targets only those compounds able to undergo electrochemical oxidation or reduction,[17] or by gas chromatography - mass spectrometry (GC-MS).[18] On the other hand, GC-MS, LC-MS and ultra performance liquid chromatography (UPLC)-MS can be used in an untargeted fashion, as can NMR spectroscopy. In an untargeted approach, the detector is used in an unbiased fashion, and without prior selection of what sort of metabolites are to be analysed. This mode has the advantage in exploratory studies of being less likely to miss unexpected metabolites.

The choice of metabolite detection system is often dependent upon the sensitivity required, or the long-term stability of the system and its throughput. Mass spectrometry is significantly more sensitive than NMR spectroscopy, which is a big advantage for some studies. On the other hand, the sensitivity of NMR spectrometers has risen dramatically in the past ten years with the advent of cryocooled probes, miniaturised detection systems and effective and efficient pulsed field gradient-based experiments.[19] In addition, NMR spectrometers tend to be more stable than mass spectrometers, as there is no direct contact between the sample and the spectrometer; the sample is held inside a glass tube or in a flow probe. By contrast, in LC-MS, for example, the eluent from the LC is injected directly into the source of the mass spectrometer and this can affect its sensitivity, resolution and general performance over time. Due to the stability and also the full automation of NMR spectrometers, the throughput of an NMR spectrometer can be very high - at least 100 samples per day, and this can be a determining factor for large, clinical studies. All of the original animal and human experiments leading to the discovery of pharmacometabonomics were conducted with NMR detection. We shall review the methodology for pharmacometabonomics experiments now, with special emphasis on the use of NMR spectroscopy. Whichever methodology is used, it should be noted that a key bottleneck is the identification of the metabolites that are found to be discriminating, or acting as biomarkers, on the basis of the data analysis. This is an area of concern in the field[20] and has been the subject of new proposals recently.[21-23]

Mass Spectrometry-Based Detection Methods. Mass spectrometers are typically used as detectors hyphenated in an online mode with a separation technology, such as gas chromatography or liquid chromatography, to give a GC-MS or LC-MS system respectively. Mass spectrometers measure the mass-to-charge ratio of charged molecules or molecular fragments following the ionisation of individual molecules as they emerge from the separation technique used. Mass spectrometry is a high 
sensitivity technique that is capable of detecting a wide array of metabolite types with high precision. In GC-MS there is a requirement that the metabolites must be volatile enough, and stable enough, to pass through the heated separation column of the gas chromatograph. In order to effect this, it is typical to extract metabolites from a biofluid and then derivatise them with a chemical agent that makes them volatile. These procedures obviously have some limitations, as not all metabolites will be extracted and/or derivatised with equal efficiency. GC-MS is typically done with electron impact (EI) ionisation of the analytes as they leave the GC and enter the source of the MS. This has the advantage that fragmentation information is available from the EI mass spectra, giving the opportunity to identify unknown molecules, and in addition, large libraries of EI mass spectra of standards are available to help confirm the identities of known compounds. However, sometimes the molecular ion is not observed and this can lead to confusion in the identification of novel metabolites whose spectra are not in the databases.[21] LC-MS methods typically use electrospray ionisation, in an atmospheric pressure source, to ionise the eluent from an HPLC (high performance liquid chromatography) system. The ionisation can either be in positive-ion mode, producing protonated molecular ions $(\mathrm{M}+\mathrm{H})^{+}$, or in negative-ion mode, producing deprotonated molecular ions (M-H)- Electrospray ionisation is a soft ionisation method, so little fragmentation is usually observed and the protonated or deprotonated molecular ions are usually the largest peaks in the spectra. The advantage of LC-MS over GC-MS is that no extraction or derivatisation processes are required; a variety of metabolites can be analysed directly from the HPLC flow. The disadvantage is that the peak widths are relatively broad and the resolution of the metabolites may not be optimal. This resolution issue has been significantly addressed with the advent of ultra performance LC (UPLC) however.[21] A further disadvantage to most MS techniques is that multiple species may be observed for each metabolite. For instance, in GC-MS studies employing derivatisation by trimethylsilylation, it is possible to form derivatives with different numbers of trimethylsilyl groups. In LC-MS studies, although no derivatisation is used, the soft ionisation can lead to the formation of a variety of molecular ion adducts including those involving sodium ions, potassium ions and water molecules.[21]

NMR Spectroscopy-Based Detection Methods. NMR Spectroscopy is the most powerful method for the elucidation of the molecular structures of compounds in the solution state. The original experiments in pharmacometabonomics were all done with NMRbased detection and we will therefore focus more on this technology in this review. NMR Spectrometers measure the signals from the nuclei of the isotopes of certain atoms, such as hydrogen-1 $\left({ }^{1} \mathrm{H}\right)$ in metabolites, as those nuclei relax after radiofrequency excitation in a strong magnetic field. NMR Spectroscopy is typically run without hyphenation to a separation system but LC-NMR and LC-NMR-MS systems can be, and have been, used in metabonomics.[24] NMR spectrometers suitable for metabonomics and pharmacometabonomics experiments are generally available with a range of magnetic field strengths from 9.4 to 18.8 Tesla, equivalent to operating at radiofrequencies between $400 \mathrm{MHz}$ and $800 \mathrm{MHz}$ respectively, for ${ }^{1} \mathrm{H}$ NMR. Operating at increased field strength has several advantages including: 1) improved sensitivity of detection; 2) increased dispersion, or spreading out of the crowded metabolite signals. The isotopes of many important elements are NMR - detectable but the most important nuclei for metabonomics are ${ }^{1} \mathrm{H}$ and ${ }^{13} \mathrm{C}$. 
NMR Spectroscopy is an incredibly powerful technology for metabolite detection and identification and has a number of key attributes:

1. ${ }^{1} \mathrm{H}$ NMR spectroscopy is the most usual form of NMR for the study of chemical or biological samples, as it has one of the highest sensitivities for detection and hydrogen atoms are ubiquitous in chemistry and in nature

2. for ${ }^{1} \mathrm{H}$ NMR, every chemically-distinct hydrogen atom in a molecule will exhibit an NMR signal at its own distinct frequency, or chemical shift, which sensitively reflects the precise, three-dimensional chemical environment of that hydrogen.

3. in ${ }^{1} \mathrm{H}$ NMR spectra, the signals of hydrogen atoms that are separated by between 2 and 3 chemical bonds (generally) exhibit 'coupling' to one another, as the magnetic state of each hydrogen nucleus affects the signals of its neighbours. Each coupling will split the signal of the coupled hydrogen into two - this type of signal is called a doublet. A hydrogen with two different neighbours will have its signal split into 4 lines - a doublet of doublets. This spin-spin coupling, or Jcoupling, gives NMR spectroscopy enormous power to piece together the structures of molecules on the basis of the coupling connectivities observed in the NMR spectra.

An example of a ${ }^{1} \mathrm{H}$ NMR spectrum of the urine of a diabetic patient is shown in Figure 1.

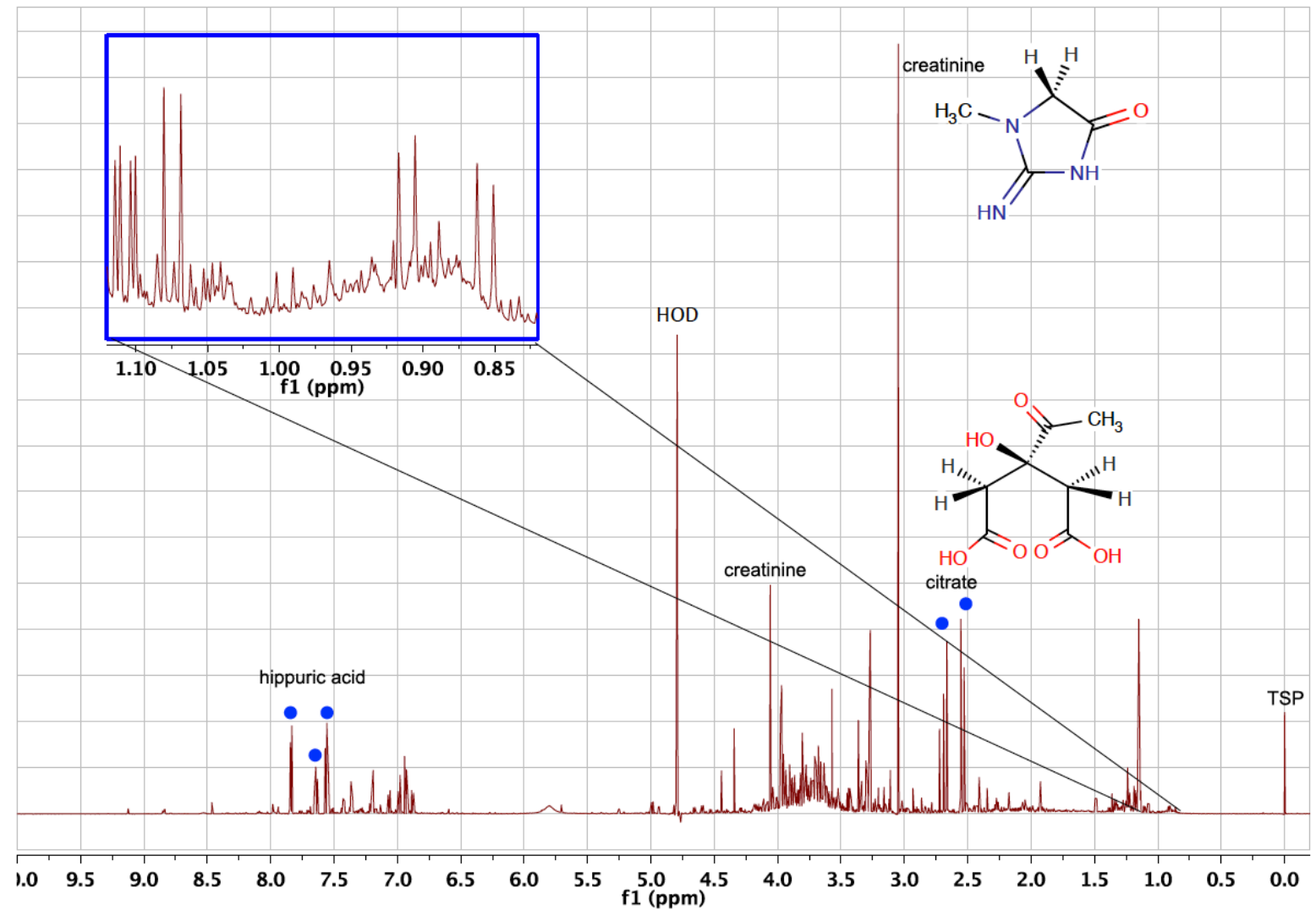

Figure 1: the $600 \mathrm{MHz},{ }^{1} \mathrm{H}$ NMR spectrum of the urine of a diabetic patient. The vertical scale is signal intensity; the horizontal scale is chemical shift in parts per million (ppm) against the reference compound trimethylsilylpropionate (TSP) which gives a singlet signal at $0 \mathrm{ppm}$. The signals of some other human (creatinine, citrate, molecular structures inset) and microbiome-derived metabolites are indicated, 
together with the residual signal from the water peak at ca $4.8 \mathrm{ppm}$ which has been largely suppressed. The positions of the three multiplet signals from hippuric acid and the two doublets of citrate are marked with filled circles. The inset box is an expansion of the region of the spectrum from 0.82 to $1.12 \mathrm{ppm}$.[25]

Figure 1 displays many of the features described above, and contains the ${ }^{1} \mathrm{H}$ signals of a vast array of metabolites. Some signals, such as those from the $\mathrm{CH}_{3}$ and $\mathrm{CH}_{2}$ groups in creatinine (see molecular structure on Figure 1), where there are no hydrogen atom neighbours within 3 chemical bonds, resonate as singlets, whereas the non-equivalent $\mathrm{CH}_{2}$ hydrogens in citrate resonate as doublets, as they couple with one another over two bonds. The inset box expansion is remarkable: it represents $<3 \%$ of the spectrum displayed and shows the signals from a large number of metabolites invisible before expansion. To determine the structures of these metabolites, two-dimensional (2D) NMR spectroscopy methods can be used, where information about the spectrum can be displayed over two frequency axes instead of one. We will not going into the details of 2D NMR methods here as excellent reference texts are available.[26]

The identification of the origin of the two doublet signals at chemical shifts ca 0.91 and ca $0.86 \mathrm{ppm}$ revealed in the expansion of Figure 1 (signals 1 and 2 respectively in Figure 2 below) is a useful case study. The signals appear to be of the same intensity: could they be from the same molecule? This question is answered by the $2 \mathrm{D}{ }^{1} \mathrm{H}$ COSY (Correlated SpectroscopY) NMR spectrum of the same urine sample (Figure 2).

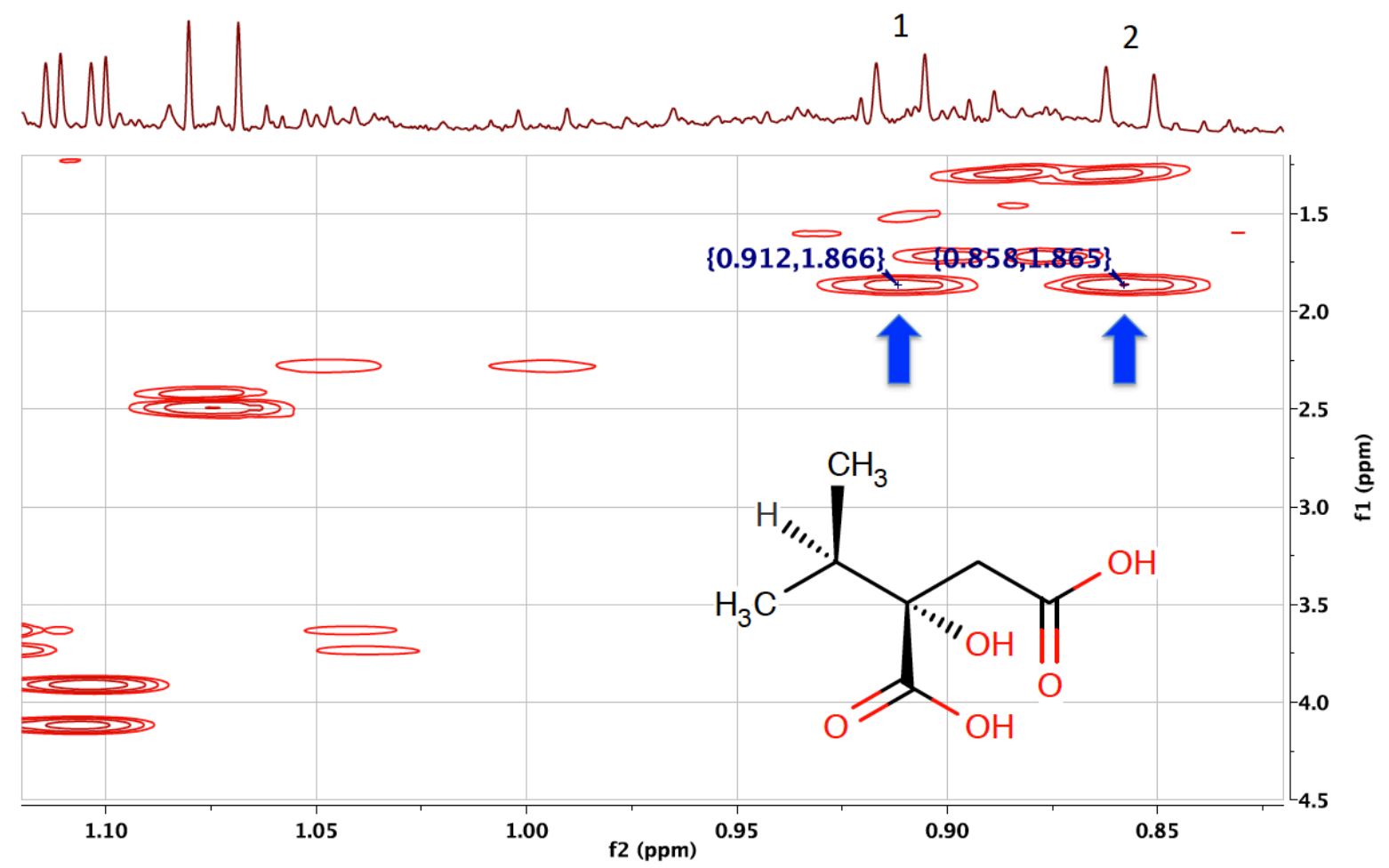

Figure 2. an expansion of the $600 \mathrm{MHz}{ }^{1} \mathrm{H}$ NMR spectrum of the urine from a diabetic patient plotted above a contour-plot representation of the 2D ${ }^{1} \mathrm{H}$ COSY (Correlated SpectroscopY) NMR spectrum of the same sample. In this experiment 'cross-peaks' occur at the intersections of the chemical shifts of spincoupled hydrogen neighbours. The arrows point to two cross-peaks showing that signals 1 and 2 are each 
spin-coupled to the same hydrogen signal resonating at a chemical shift of ca $1.865 \mathrm{ppm}$. The molecular structure of the metabolite isopropylmalate, whose $\mathrm{CH}_{3}$ groups give rise to signals 1 and 2 is shown.[25]

As signals 1 and 2 are doublets, they must be coupled to just one neighbouring hydrogen atom each. From their chemical shift, these signals are almost certainly methyl groups, $\mathrm{CH}_{3}$; thus we know we have two $\mathrm{CH}_{3}-\mathrm{CH}$ units. However, in Figure 2, it is clear from the cross peaks marked with arrows that both signals 1 and 2 are coupling to the same hydrogen, one that resonates at ca $1.865 \mathrm{ppm}$. Thus, we can deduce that we have a $\mathrm{CH}_{3}-\mathrm{CH}-\mathrm{CH}_{3}$, or isopropyl moiety in the metabolite. Searching metabolite databases such as the Human Metabolome Database (HMDB),[27] we find that the known metabolite isopropylmalate, (see Figure 2) has almost identical NMR shifts and coupling constants, and after some further confirmation that the other parts of the molecule were present, this metabolite was conclusively identified. This gives a glimpse into the tremendous power of NMR to identify unknown molecules in biological fluids.

There are additional features of NMR that make it very attractive as an analytical tool for the study of biological fluids. Firstly, ${ }^{1} \mathrm{H}$ NMR spectroscopy is inherently fully quantitative (given certain provisos) and the signal area of a methylene group $\left(-\mathrm{CH}_{2}-\right)$ in a molecule will be double that of a methyne group, $\mathrm{CH}$, in the same molecule. In the same way of course, the signal area of the $\mathrm{CH}_{3-}$, or methyl group in lactate, will be double that of the signal area of the $\mathrm{CH}_{3}$ - group of alanine in a ${ }^{1} \mathrm{H}$ NMR spectrum of human urine, if the concentration of lactate is twice that of alanine in the urine.

Secondly, NMR spectroscopy is an 'open-window' detector that gives signals for any molecules that are present in a sample above the detection limit; this is unlike many other detection technologies which have to be set up specifically for the observation of only certain classes of compounds.

Thirdly, NMR spectroscopy does not consume any sample in the course of the analysis and also does not usually perturb any chemical or biochemical equilibria occurring in the sample.

Statistical Analysis of Metabolite Profiles. In a human pharmacometabonomics experiment, the metabolic profiles of the biofluids of a large number of subjects will be measured before and after drug administration. The analysis of the vast amount of data generated will require the assistance of computers. For urine in particular, the spectral data are generally normalised so that variations in urine dilution due to variable water consumption, and then excretion, do not confound the analysis. The biofluid spectra will also be scaled so that the variation of the intensities of large peaks does not swamp potentially important, but smaller, variations in the intensities of smaller signals. Finally, for NMR-based detection, the signals in the spectra will need alignment so that small differences in resonance position, due to small changes in $\mathrm{pH}$ or ionic strength between samples, do not lead to the misclassification of signals. All of these procedures are now well defined, even if no universally agreed protocols have yet emerged.[28-30]

The analysis of the pre-dose biofluid spectra in pharmacometabonomics experiments by multivariate statistical projection methods, can be done by both unsupervised methods and supervised methods.[31] In the unsupervised methods, such as principal component analysis (PCA), no information is given to the system about post-dose class 
membership of the subjects e.g. good responders or poor responders to the drug. The PCA seeks to determine, from an unbiased analysis of the pre-dose spectral data alone, if there is information that can distinguish between the post-dose responders and nonresponders. PCA is essentially a dimension-reduction methodology, where linear combinations of the input variables (pre-dose spectral intensity values) are used to compute principal components, each orthogonal to one another, that are optimised to best describe the variation amongst the data. It is typical to use each spectral data point as an input variable in this way, thus resulting in may thousands of such variables. The first principal component, PC1, describes the most variance in the data, with each successive principal component, PC2, PC3, PC4 etc describing less and less of the variation. The resulting PCA can be plotted as a scores plot, which shows the position of each sample across two or more of the principal components. A conceptual example is shown in 3. The loadings plot of the PCA can be used to determine which variables in the pre-dose data contributed most to the discrimination between the post-dose properties of the samples. These unsupervised methods are thus free from bias and potential over-fitting of data.

In supervised methods, such as projection to latent structures (PLS), information is provided to the system about the post-dose response of the subjects, in order to aid the pre-dose analysis. The information provided can either be categorical, such as good responder vs non-responder, or quantitative, such as the value for some key post-dose biomarker, such as blood pressure. The supervised multivariate analysis methods then seek to find discriminating variables in the pre-dose spectral data that can explain the variation observed in the post-dose data. The methods have evolved significantly in recent years and include procedures to eliminate non-discriminating variables, such as orthogonal, or 0-PLS. The danger with these methods is of over-fitting, especially when there are large numbers of variables to consider (see above), so care must be paid to validation of the findings and to ensuring that the false discovery rate is low.

Figure 3 illustrates in a conceptual fashion the differences between metabonomics experiments and pharmacometabonomics experiments.

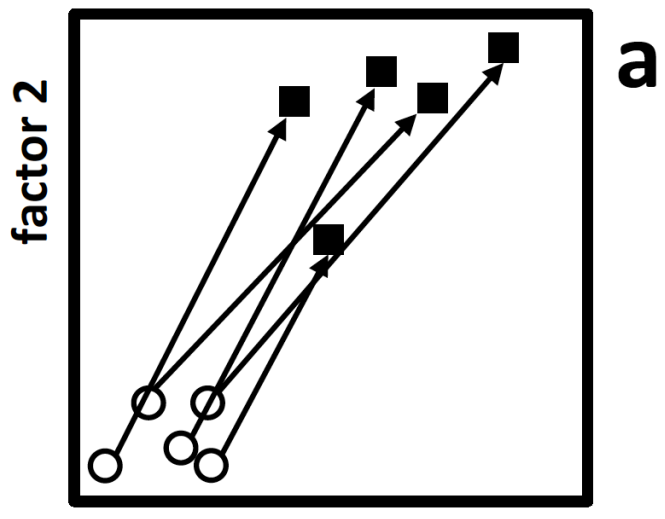

factor 1 metabonomics

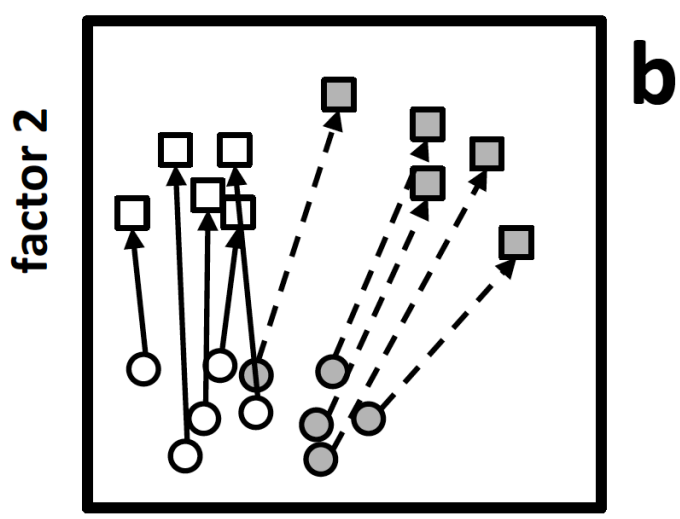

factor 1 pharmacometabonomics 
Figure 3: a conceptual view of the differences between metabonomics and pharmacometabonomics experiments as illustrated by multivariate analysis e.g. PCA where factors 1 and 2 would be the first and second principal components in a PCA scores plot (see text). In a metabonomics experiment, the pre-dose metabolic state of the subjects (open circles) is changed by an intervention, such as drug dosing, to a different post-dose metabolic state (closed squares). The arrows portray the metabolic trajectories of the subjects that occur due to the intervention. In a pharmacometabonomics experiment, one sub-group of the subjects has a different pre-dose metabolic state or phenotype to another (grey circles vs open circles). Following drug dosing, the subjects have different responses to the drug according to their different pre-dose states and different metabolic trajectories are observed for the two sub-groups. An analysis of the pre-dose metabolic profiles would, in this case, be able to predict (albeit not perfectly because of some group overlap) the post-dose outcome: this is pharmacometabonomics.

\section{Clinical Pharmacometabonomics}

Following the discovery of pharmacometabonomics in animals,[16] it became important to determine if pharmacometabonomics would work in humans. A clinical trial involving human volunteers was therefore designed by a joint Pfizer - Imperial College team led by Jeremy Everett and Jeremy Nicholson. The aim of this trial in 100 fit and healthy male volunteers was to establish if pre-dose urine metabolite profiles could predict the metabolism of the analgesic drug paracetamol (acetaminophen) after a normal clinical dose of two $500 \mathrm{mg}$ tablets.[32] This ethically approved study was conducted in 2003. Paracetamol was chosen for this study as it is metabolised principally to sulphate and glucuronide metabolites (Figure 4) and significant interindividual differences in the ratios of these metabolites has been observed.



Figure 4. The chemical structures of paracetamol and its major human metabolites.

The trial protocol did not stipulate a standard diet but did place restrictions on the volunteers in terms of not taking drugs, herbal remedies or dietary supplements prior to sample collection, which was conducted pre-dose and 0 to 3 hours and 3 to 6 hours post-dose. Interestingly, ${ }^{1} \mathrm{H}$ NMR spectroscopy is so powerful that it was able to spot the volunteers who had not obeyed these instructions! The $600 \mathrm{MHz}^{1} \mathrm{H}$ NMR spectra of the pre-dose and post-dose urines from two of the volunteers on this clinical trial are shown in Figure 5. 




Figure 5: $600 \mathrm{MHz}{ }^{1} \mathrm{H}$ NMR spectra of the urine from two different human volunteers. A and B: Pre-dose and 0 to 3 hour post-dose spectra from volunteer 1 . The main differences between the pre-dose and the post-dose spectra are the appearance of signals from paracetamol and its metabolites in the aromatic region, 7.0 to $8.0 \mathrm{ppm}$, and in the acetyl region, 2.14 to $2.20 \mathrm{ppm}$. The insets are expansions of those regions. $C$ and $D$ : corresponding pre-dose and post-dose spectra for volunteer 2 . Key to peak numbers: 1 , creatinine; 2 , hippurate; 3 , phenylacetylglutamine; 4 , metabolite 4 (unknown at the time: see text); 5 , citrate; 6 , cluster of $N$-acetyl groups from paracetamol-related compounds; 7, paracetamol sulfate; 8, paracetamol glucuronide; 9, other paracetamol-related compounds. Reproduced from PNAS.[32] 
The ${ }^{1} \mathrm{H}$ NMR signals of both the acetyl, $\mathrm{CH}_{3}-\mathrm{CO}$, and the para-disubstituted aromatic protons of the two key drug metabolites, paracetamol sulphate (S), 7, and paracetamol glucuronide $(G), 8$, are clearly visible in the post-dose spectra. In addition, it is clear that the ratios of $S / G$ in these two, 0 to 3 hour post-dose urines are different, with a much larger S/G ratio for volunteer 2 (Figure 5D), that for volunteer 1 (Figure 5B). A considerable amount of time was spent in computer-based statistical pattern recognition analyses of all the 300 NMR spectra from this study but with little significant output. It is astounding that the key pre-dose biomarker signal is actually visible in the figure but was not found by these methods. The pre-dose urine ${ }^{1} \mathrm{H}$ NMR spectrum of volunteer 1 (Figure 5A) shows both a singlet signal at $2.35 \mathrm{ppm}$ and a pair of doublets between 7.2 and $7.3 \mathrm{ppm}$, from a metabolite labeled 4, that were not clearly present in the pre-dose urine of volunteer 2 (Figure 5C). Further analysis found that high pre-dose levels of metabolite 4 were correlated with low post-dose ratios of paracetamol sulphate to paracetamol glucuronide (S/G), as seen in Figure 5. This effect is clearly shown in Figure 6.
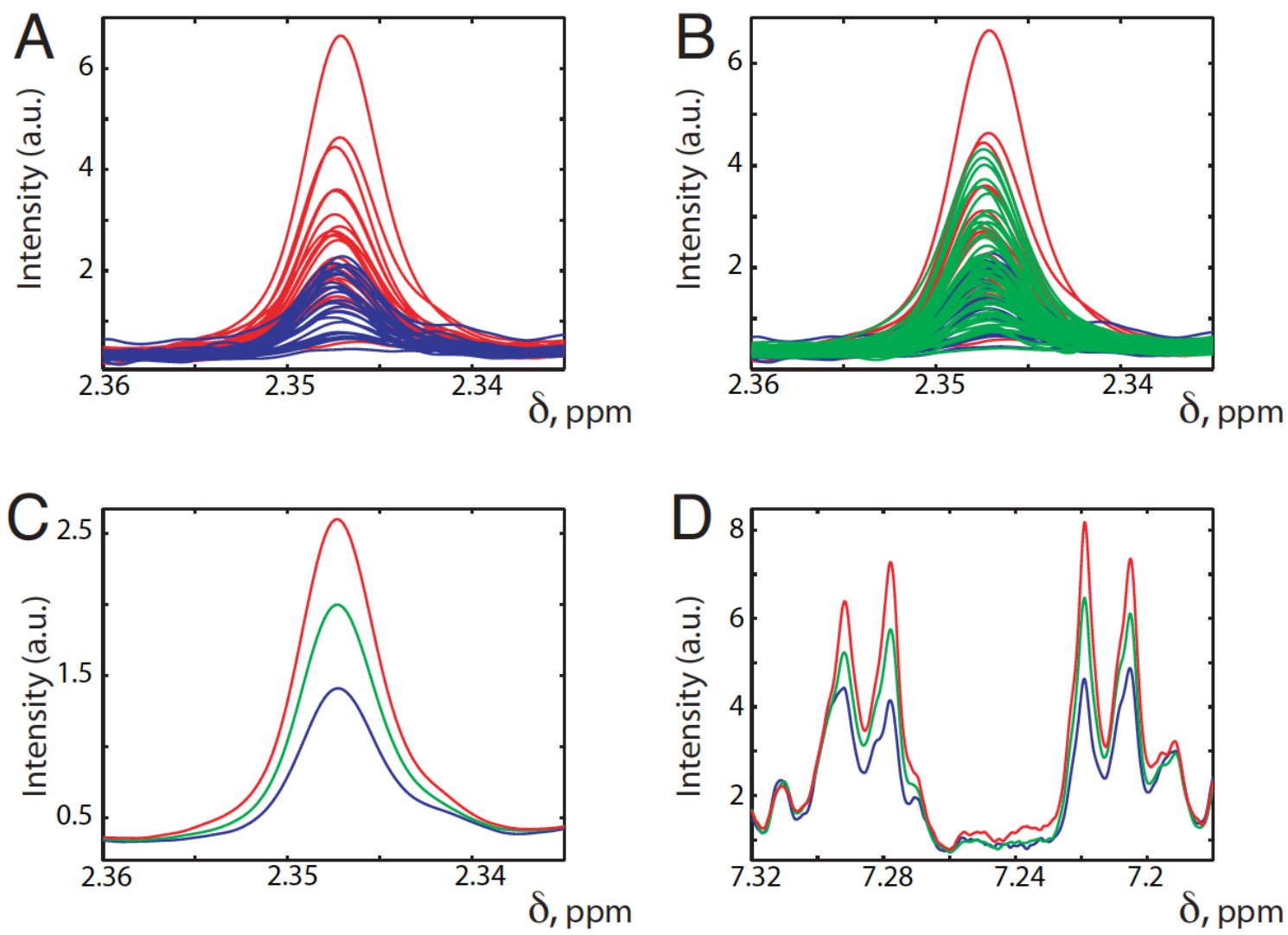

Figure 6: A series of $600 \mathrm{MHz}{ }^{1} \mathrm{H}$ NMR spectra of the pre-dose urines of human volunteers subsequently dosed with a standard $1 \mathrm{~g}$ oral dose of paracetamol. A: The NMR spectra of the singlet $\mathrm{CH}_{3}$ peak of metabolite 4, colour-coded for the 25 volunteers with the highest (blue spectra), and the 25 volunteers with the lowest (red spectra), ratios of paracetamol sulfate to paracetamol glucuronide (S/G) in their post-dose urine. B: as for $\mathrm{A}$, but with the addition of the pre-dose spectra for 49 additional volunteers with intermediate (green spectra) post-dose $\mathrm{S} / \mathrm{G}$ ratios. C: The same information as in $\mathrm{B}$, but with a single averaged pre-dose spectrum shown for each of the three groups. D: The corresponding aromatic region of the pre-dose ${ }^{1} \mathrm{H}$ NMR spectra of metabolite 4 with the sample post-dose colour-coding as in B and C. Reproduced from PNAS.[32] 
It is clear from Figure 6 that there is a relationship of some sort between the levels of metabolite 4 in the pre-dose urine and the post-dose urinary ratios of paracetamol sulphate to paracetamol glucuronide (S/G). This is most clearly displayed in Figure 7, which shows the relationship between the levels of metabolite 4, normalized to creatinine, and the $S / G$ ratio of all volunteers.



Figure 7. The relationship between post-dose paracetamol sulfate to paracetamol glucuronide (S/G) ratio and the pre-dose ratio of metabolite 4 normalised to creatinine in the urines from human volunteers on a paracetamol trial. Adapted from PNAS.[32]

Two things are clear from Figure 7. Firstly, if the pre-dose ratio of metabolite 4 normalised to creatinine is high ( $>0.06$ ), then the post-dose $\mathrm{S} / \mathrm{G}$ ratio is always low (< $0.8)$. Secondly, if the pre-dose ratio of metabolite 4 to creatinine is low, then no predictions of the post-dose $S / G$ ratio are possible. A Mann-Whitney $U$ test in conjunction with a Bonferroni correction of 100, to counter the effects of multiple hypothesis testing, showed that this relationship was significant at the $5 \%$ level: the raw $p$ value was $1.0 \times 10^{-4}$. A value lower that $5 \times 10^{-4}$ is required for statistical significance at the $95 \%$ confidence level with a correction factor of 100 . A similar, statistically significant relationship was found between the corresponding 3 to 6 hour post-dose S/G ratio and the pre-dose metabolite 4 to creatinine ratio (data not shown, $p$ $=1.2 \times 10^{-4}$ ).

It thus became imperative to determine the structure of metabolite 4 , which had become a pre-dose pharmacometabonomic biomarker for post-dose drug metabolism outcome. The singlet nature of the methyl group of metabolite 4 , showed that it was attached to a carbon atom with no hydrogens on it. The chemical shift of the methyl 
group, indicated that it was attached to an sp2 hybridised carbon, probably an aromatic carbon atom. Given the co-occurrence of the aromatic signals, which indicated that the ring was para-disubstituted, it was postulated that metabolite 4 had the structure shown in Figure 8.

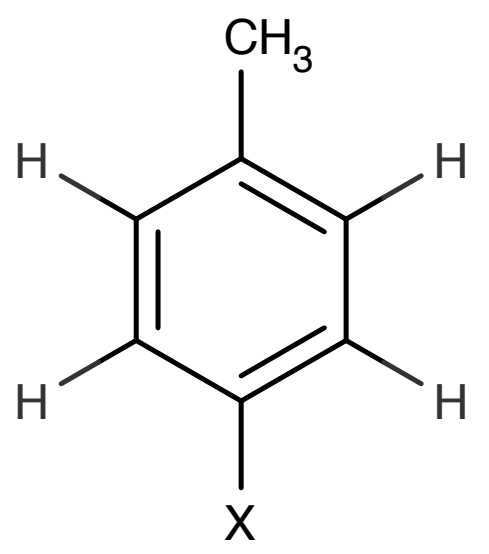

Figure 8: a partial structure of metabolite 4, deduced from the pre-dose ${ }^{1} \mathrm{H}$ NMR spectra of the urine from the volunteers on the paracetamol pharmacometabonomics trial. Substituent X is an unknown moiety.

The nature of substituent $X$ was deduced when incubation of a sample of pre-dose urine with sulphatase enzyme led to the decrease in the signals of metabolite 4 and the emergence of new signals ascribed to para-cresol (4-methylphenol), by comparison with an authentic reference standard. Confirmation that metabolite 4 was para-cresol sulphate was obtained by synthesis of the molecule from authentic para-cresol and spiking the synthetic material into a pre-dose urine sample.

The identification of metabolite 4 as para-cresol sulphate, was a shock as it is not a human metabolite in origin, deriving from the sulphation of para-cresol. Most paracresol in the human body is thought to originate from bacteria in the gut microbiome and from Chlostridia species in particular.[32] It is thus now apparent that the metabolism of paracetamol in humans - one of the most studied drugs in the world - is not fully under human control. The hypothesis to explain this result is as follows. If the gut microbiome of a person is excreting large quantities of para-cresol, the body will want to eliminate this toxic molecule rapidly. Unlike rats and mice that metabolise paracresol by both glucuronidation and sulphation, humans metabolise para-cresol largely by sulphation. This can put a stress on a person's sulphation system, which does not have a large capacity, especially if the person subsequently takes a dose of a drug that requires sulphation for its metabolism, such as paracetamol. In this case, both paracetamol and para-cresol are in competition for the same human sulphatase enzymes and for the same enzyme co-factor, 3'-phosphoadenosine 5'-phosphosulfate (PAPS, see Figure 9). Thus a person whose sulphation capacity is already reduced by virtue of a gut microbiome-derived sulphation load from para-cresol production, will switch the metabolism of paracetamol from sulphation to glucuronidation. On the other hand, in the absence of a significant microbiome-derived sulphation load, a person will metabolise paracetamol across both the sulphation and glucuronidation pathways in a fashion that depends on a number of genetic and environmental parameters, and that is not currently predictable. 
This clinical trial in volunteers represents the first demonstration of pharmacometabonomics in humans and the first demonstration of the use of pre-dose metabolic profiles to predict drug metabolism in humans.[32] The initial publication was accompanied by a commentary by Ian Wilson.[33] It is satisfying that the simple biomarker associated with the prediction of the metabolism has a logical connection to the drug metabolism itself. It should be noted however, that the finding of the linkage between the pre-dose levels of para-cresol sulphate and the post dose sulphation of a drug, will not be limited to application to paracetamol. This finding should hold for any high dose drug that requires the body to drive a significant metabolic flux through the sulphation pathway. It is also worth noting that there are many endogenous pathways involving sulphation steps that could also be affected by this phenomenon. Finally, it should be noted that there are many human diseases such as childhood autism, childhood hyperactivity and Parkinson's Disease that are associated either with elevated para-cresol levels, or decreased S/G ratios after the dosing of paracetamol, implying impaired sulphation and altered microbiomes in these disease states.

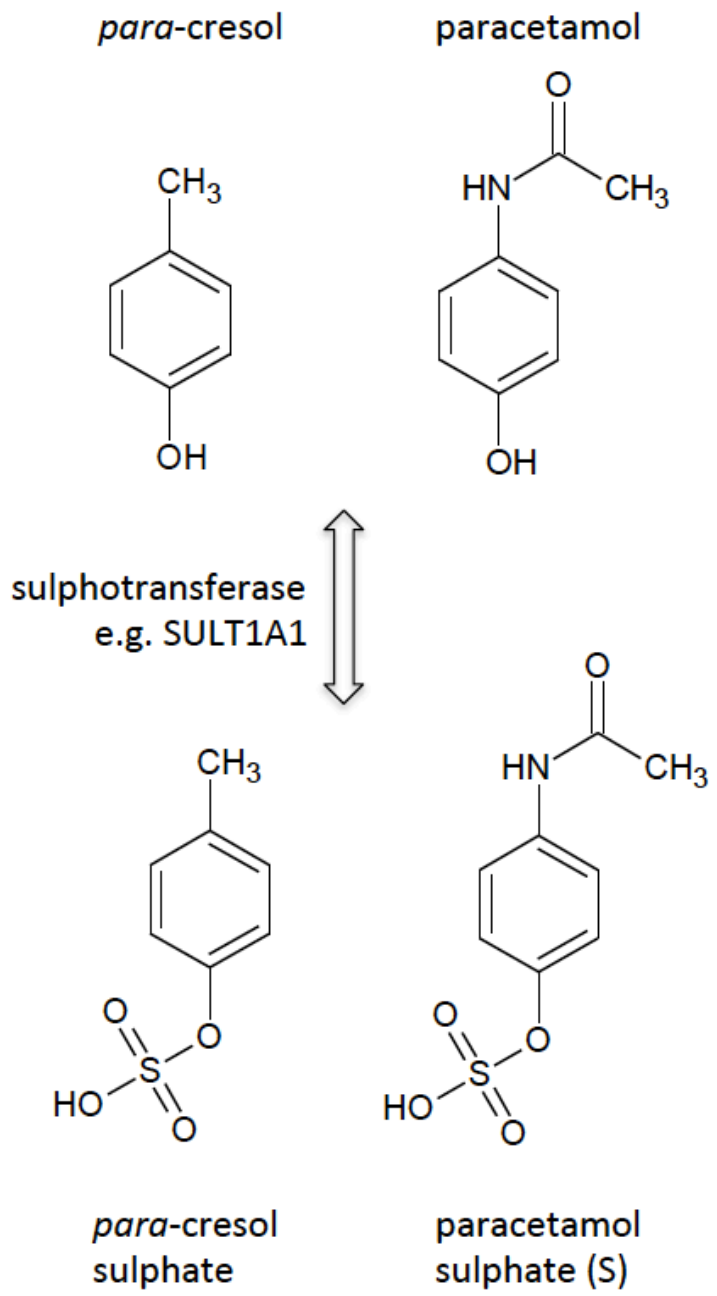

Figure 9. The molecular structures of para-cresol and paracetamol and their sulphation by sulphotransferase enzymes such as SULT1A1. 


\section{Progress in Clinical Pharmacometabonomics}

Progress has been rapid since the publication of the initial discovery of pharmacometabonomics in humans in 2009 and the topic has been reviewed several times.[34-37] In this work we will focus on clinical demonstrations of pharmacometabonomics. Table 1 lists all the studies found by searching for the terms pharmacometabonomics, pharmacometabolomics, pharmaco-metabonomics and pharmaco-metabolomics in October 2014 using Web of Science, Pub Med and other search facilities.

\begin{tabular}{|c|c|c|}
\hline class of experiment & study & authors and reference \\
\hline $\begin{array}{l}\text { prediction of } \\
\text { pharmacokinetics }(\mathrm{PK})\end{array}$ & prediction of Tacrolimus PK & Yoon and Hwang [38] \\
\hline \multirow[t]{3}{*}{$\begin{array}{l}\text { prediction of drug } \\
\text { metabolism }\end{array}$} & $\begin{array}{l}\text { prediction of metabolism of } \\
\text { paracetamol / acetaminophen }\end{array}$ & Clayton et al [32] \\
\hline & prediction of CYP3A4 induction & Rahmioglu et al [39] \\
\hline & prediction of CYP3A activity & Shin et al [40] \\
\hline \multirow[t]{5}{*}{$\begin{array}{l}\text { prediction of drug } \\
\text { efficacy }\end{array}$} & $\begin{array}{l}\text { prediction of simvastatin } \\
\text { efficacy in patients on the } \\
\text { Cholesterol and } \\
\text { Pharmacogenomics study }\end{array}$ & $\begin{array}{l}\text { Kaddurah-Daouk et al } \\
{[18,41] \text { and Trupp et al }[42]}\end{array}$ \\
\hline & $\begin{array}{l}\text { prediction of citalopram/ } \\
\text { escitalopram response in } \\
\text { patients with major depressive } \\
\text { disorder (MDD) }\end{array}$ & $\begin{array}{l}\text { Ji et al [43] and Abo et al } \\
{[44]}\end{array}$ \\
\hline & $\begin{array}{l}\text { prediction of sertraline and } \\
\text { placebo responses in patients } \\
\text { with MDD }\end{array}$ & $\begin{array}{l}\text { Kaddurah-Daouk et al, } \\
{[17,45] \text { Zhu et al }[46]}\end{array}$ \\
\hline & $\begin{array}{l}\text { prediction of efficacy of anti- } \\
\text { psychotics }\end{array}$ & Condray et al [47] \\
\hline & $\begin{array}{l}\text { prediction of response to } \\
\text { aspirin }\end{array}$ & $\begin{array}{l}\text { Lewis et al [48] and Ellero- } \\
\text { Simatos et al [49] }\end{array}$ \\
\hline \multirow[t]{3}{*}{$\begin{array}{l}\text { prediction of adverse } \\
\text { events }\end{array}$} & $\begin{array}{l}\text { prediction of weight gain in } \\
\text { breast cancer patients } \\
\text { undergoing chemotherapy }\end{array}$ & Keun et al [50] \\
\hline & $\begin{array}{l}\text { prediction of toxicity in patients } \\
\text { with inoperable colorectal } \\
\text { cancer treated with } \\
\text { Capecitabine }\end{array}$ & Backshall et al [51] \\
\hline & $\begin{array}{l}\text { prediction of toxicity of } \\
\text { paracetamol / acetaminophen } \\
\text { ('early-onset } \\
\text { pharmacometabonomics') }\end{array}$ & Winnike et al [52] \\
\hline
\end{tabular}




\begin{tabular}{|l|l|l|}
\hline & \\
\hline $\begin{array}{l}\text { predictive } \\
\text { metabonomics }\end{array}$ & $\begin{array}{l}\text { prediction of future impaired } \\
\text { glucose tolerance or Type-2 } \\
\text { diabetes onset }\end{array}$ & Wang-Sattler et al [53] \\
\hline & & \\
\hline
\end{tabular}

Table 1. An overview of the human pharmacometabonomics and predictive metabonomics studies reported in the literature to late 2014 .

In the first pharmacometabonomics study with patients, as opposed to human volunteers, Keun et al[50] demonstrated that pre-dose serum levels of lactate, alanine and $\%$ body fat were predictive of weight gain in a group of 21 breast cancer patients undergoing 5-fluorouracil-, cyclophosphamide- or epirubicin-based i.v. chemotherapy. Since weight gain in these patients is a known risk factor for disease recurrence, it is hoped that this predictive test will be useful to patients in this setting.

For drugs with a narrow therapeutic index, the prediction of PK can be important. Yoon and Hwang[38] showed that LC-MS analysis of the pre-dose urinary levels of cortisol, 1methylguanosine, acetylarginine and phosphoethanolamine in 29 volunteers could be used to classify them into high, medium and low, area under the curve (AUC) groups, following oral dosing with Tacrolimus, an immunosuppressant agent. An additional model was built to predict drug clearance. The authors envisaged that they would be able to predict post-transplant pharmacokinetics of Tacrolimus using this approach.

In a study that was not strictly pharmacometabonomics, Winnike and co-workers[52] returned to the study of paracetamol in volunteers, but this time, instead of predicting the metabolism of the drug, they used a pharmacometabonomics approach to attempt to predict the susceptibility of 71 healthy male and female volunteers to the toxic effects of a high dose of paracetamol: $4 \mathrm{~g}$ daily for a week. The study failed to reach statistical significance for the prediction of the onset of toxicity based on the analysis of pre-dose urine metabolite profiles. However, it was found that individual susceptibility to reversible liver injury (measured by increased serum alanine aminotransferase (ALT) levels) could be predicted from an NMR analysis of urine metabolite profiles shortly after dosing, and well before any changes in ALT levels had been observed. The discrimination was based on the levels of the toxic metabolite $N$-acetyl paraquinone imine (NAPQI) plus some other endogenous metabolites. This was termed 'earlyintervention pharmacometabonomics' and it was envisaged that such an approach might have applicability in other cases where a strictly pharmacometabonomics approach does not work.

Three separate papers from Kaddurah-Daouk et al have been published of pharmacometabonomics predictions of statin efficacy from patients on the Cholesterol and Pharmacogenetics (CAP) study. In the first study,[41] 36 good responders and 36 poor responders to simvastatin therapy were chosen for study. The pre-dose plasma levels of the phosphatidylcholine metabolite PC18:2n6, the cholesterol ester CE18:1n7 and the free fatty acid FA18:3n3, amongst other molecules, were found to be predictive of post-dose efficacy as measured by reduction in patient low density lipoproteincholesterol (LDL-C) reduction. A targeted GC-MS and LC-MS study of patients from the same CAP study was reported later.[18] Pre-dose plasma levels of taurocholic acid (TCA), glycocholic acid (GCA), taurochenodeoxycholic acid (TCDCA), 
glycochenodeoxycholic acid, (GCDCA) and glycoursodeoxycholic acid (GUDCA), were found to be negatively correlated with post-dose \% LDL-C reduction in 100 patients representing the full range of patient responses. Pre-dose levels of two bile acids, chenodeoxycholic acid (CDCA) and deoxycholic acid (DCA), were positively correlated to post-treatment simvastatin levels in this same full-range patient subset. Pre-dose levels of the secondary bile acids lithocholic acid (LCA), taurolithocholic acid (TLCA), and glycolithocholic acid (GLCA), as well as coprostanol (COPR), all produced by gut bacteria, were shown to be positively correlated to post-dose \% LDL-C reduction in a separate group of 24 good and 24 poor responders to simvastatin. It had previously been reported that a single nucleotide polymorphism (SNP), rs4149056, in the gene encoding the organic anion transporter SLC01B1 was correlated with plasma levels of simvastatin, and that the transporter played a role in the hepatic uptake of bile acids in addition to simvastatin. In an interesting example of the combined use of genetic and metabolic information, it was hypothesised that competition between bile acids and simvastatin for this transporter may affect both the PK and efficacy of simvastatin. A third pharmacometabonomics paper on the same patient group used GC-time of flightMS detection technology to determine that greater \% LDL-C reduction in the good responder sub-group was significantly associated with higher pre-dose levels of hexaric acid and lower pre-dose levels of xanthine, 2-hydroxyvaleric acid, succinic acid and stearic acid.[42] It was concluded that clusters of metabolites from diverse pathways were modulating the response to the drug.

A series of papers from Kaddurah-Daouk et al and Weinshilboum have emerged on the use of pharmacometabonomics and pharmacometabonomics-informed pharmacogenomics to predict anti-depressant efficacy in patients with major depressive disorder. In the first paper,[43] patients were selected from the Mayo ClinicNIH Pharmacogenetics Research Network (PGRN) Citalopram/Escitalopram Pharmacogenomics (Mayo-PGRN SSRI) study. GC-MS study of the pre-dose plasma from 20 escitalopram remitters (remission defined as having a Quick Inventory of Depressive Symptomatology - Clinician Rated (QIDS-C) score of $\leq 5$ after therapy) and 20 nonremitters (defined as having a QIDS-C score of $>5$ after 8 weeks of therapy), showed that pre-dose glycine levels were significantly associated with post-dose clinical outcomes. In what was termed a pharmacometabonomics-directed pharmacogenomics study, genotyping of tagSNPs in genes involved in glycine biosynthesis and metabolism showed that the commonly occurring SNP, rs10975641, was significantly associated with citalopram/escitalopram treatment outcome. This finding was validated in a separate study of patients from the Sequenced Treatment Alternatives to Relieve Depression (STAR*D) study. The use of pharmacometabonomics to inform a pharmacogenomics study in this way is an exciting development that signposts the synergistic way in which these two areas can operate. A follow up genome-wide association study (GWAS) on the same MDD patient group largely confirmed the previous tag SNP findings.[44]

Kaddurah-Daouk and co-workers reported the prediction of response to the antidepressant sertraline, and to placebo-treatment, in a double-blind, randomised study of a cohort of 89 outpatients with a primary diagnosis of MDD by accepted criteria.[17] Targeted liquid chromatography electrochemical (LC-EC) analysis of pre-dose serum showed that the levels of dihydroxyphenylacetic acid (DOPAC), 4-hydroxyphenyllactic acid (4-HPLA), serotonin (5-HT) and gamma tocopherol, plus some unknowns, were 
responsible for a partial separation of responders from non-responders, for patients treated with sertraline. The pre-dose serum levels of hypoxanthine, xanthine and uric acid, 5-methoxytryptophol (5-MTPOL), 5-HT, 3-hydroxykynurenine (3-OHKY) and 5hydroxyindoleacetic acid (5-HIAA), DOPAC, cysteine and several tocopherols, plus some unknowns, were responsible for the partial separation of the responders and nonresponders who were treated with placebo. Thus, there was some overlap between the pre-dose metabolites that were discriminating for responses to sertraline and placebo treatments. It was noted that 5-MTPOL is produced from serotonin, 5-HT, and that 4HPLA is in a phenylalanine pathway linked to both tyrosine and catecholamine pathways. It was not noted that 5-HIAA is a serotonin metabolite that is used as a surrogate for serotonin bioassays in man.[54] A second publication reported on LC-EC and GC-TOF-MS studies on an overlapping set of patients from the same study.[46] In this study it was reported that pre-dose levels of 5-methoxytryptamine (5-MTPM), a metabolite not reported as significant in the previous study, were correlated with sertraline treatment response. In addition, the ratios of melatonin to 5-HT, melatonin to 5-MTPM, $\mathrm{N}$-acetyl 5-HT to 5-MTPM and 5-HIAA to 5-MTPM were all significantly and negatively correlated with sertraline treatment outcome. For the placebo group, the pre-dose levels of 5-MTPOL were again negatively correlated with response to treatment, as was the ratio of 5-MTPOL to tryptophan. None of the other metabolites reported previously[17] as significantly correlated with placebo response were significantly correlated in this follow-up study. A third publication on the same outpatient group, this time using GC-TOF-MS and entitled 'Pharmacometabolomic mapping of early biochemical changes induced by sertraline and placebo', actually contains metabonomics data but no pharmacometabonomics data.[45] A paper entitled 'Pharmacometabolomics Reveals Racial Differences in Response to Atenolol Treatment' from the same group [55] also contains only metabonomics data on the changes induced by the drug with no element of prediction of response to atenolol. It is hoped that the clear definition[16] of the terms pharmacometabonomics and pharmacometabolomics will not be loosened, as has unfortunately occurred with pharmacoproteomics, [34] as this will be confusing.

The group of Yao et al used LC-EC to show that low pre-dose plasma levels of 3hydroxykynurenine (3-OHKY) were predictive of greater response to treatment at week 4 , with one or more of five antipsychotic drugs, in 25, first-episode, neuroleptic-naive schizophrenia patients.[47] However, pre-dose plasma 3-OHKY levels were also negatively correlated with pre-dose levels of psychological disturbance, and it was postulated that the kynurenine pathway may influence dopaminergic as well as glutaminergic systems.

Moving from efficacy prediction to toxicity prediction, the group of Keun have also shown that the analysis of pre-dose serum from cancer patients can predict toxicity when treated with capecitabine.[51] The study included 54 patients with locally advanced or metastatic colorectal cancer. Toxicities were assessed using the National Cancer Institute Common Toxicity Criteria v2.0. In this study $600 \mathrm{MHz}{ }^{1} \mathrm{H}$ NMR spectroscopy was used to show that higher post-dose toxicity was correlated with higher pre-dose levels of low density lipoprotein-derived lipids, including polyunsaturated fatty acids and choline phospholipids. 
Two reports have appeared concerned with the prediction of the induction and activity of the cytochrome P450 (CYP) drug metabolizing enzymes. These enzymes are responsible for the metabolism of many drugs and have important influences on drug PK, efficacy and safety. They have therefore also been the subject of many pharmacogenomics studies. In the first pharmacometabonomics study, Kemsley et al reported on an NMR spectroscopy-based study of the induction of CYP3A4 in a total of 301 female twins from the TwinsUK registry, following the administration of a CYP3A4 inducer (extract of St John's Wort) for two weeks.[39] A total of 74 pairs of dizygotic twins and 47 pairs of monozygotic twins were enrolled. Each subject was then dosed with quinine (Q) on day 14, which is metabolised to 3-hydroxyquinine (3-OHQ) by CYP3A4. The ratio of 3-OHQ: Q was measured by UPLC-MS and used as an indicator of the induction of CY3A4 by the St John's Wort extract. Multiple linear regression was used to establish relationships between pre-dose urine metabolite profiles, as measured by ${ }^{1} \mathrm{H}$ NMR with a binning approach, and post-dose CYP3A4 induction, as measured by 3-OHQ: Q ratios. Nine NMR bins (small segments of the spectra) were significantly correlated with CYP3A4 induction, including bins corresponding to signals from indoxyl sulfate, guanidine acetate, glycine, scyllo-inositol, proline betaine and alanine, but no mechanistic rationale for these metabolites was clear. Further regressions were reported including two involving BMI and the UPLC-MS batch codes but the significance of using those variables as predictors is unclear.

A different approach was taken by Cho et al who used targeted GC-MS of the urines of 24 healthy, male volunteers to predict CYP3A activity as measured by plasma midazolam clearance, in a study that involved dosing the volunteers with midazolam in three distinct periods: control state, CYP3A inhibition state (additional administration of ketoconazole), and CYP3A induction state (additional administration of rifampicin).[40] The final predictive equation for midazolam clearance involved ratios of pre-dose endogenous metabolites ( $7 \beta$-dehydroepiandrosterone: dehydroepiandrosterone and $6 \beta$-hydroxycortisone: cortisone) and the level of dehydroepiandrosterone, together with the $C Y P 3 A 5^{*} 1 /^{*} 3$ genotype, and gave an $\mathrm{r}^{2}$ of 0.70 .

Variability of response to the important analgesic and anti-inflammatory drug aspirin is an issue. This was studied by Kaddurah-Daouk, Shuldiner et al in a pharmacometabonomics-informed pharmacogenomics study.[56] The first part of the study used GC-MS to analyse metabolite profiles in pre-dose and post-dose serum samples from 76 healthy Amish volunteers from the Heredity and Phenotype Intervention (HAPI) Heart Study. These volunteers were classified as good responders or poor responders to aspirin by post-dose platelet aggregation assays. No significant pharmacometabonomics findings were reported in this part of the study, but in a smaller replication set of 37 additional subjects it was reported that inosine levels were significantly higher in poor responders, both pre-dose and post-dose. Interestingly, the Supplementary Information to this paper appears to show significant differences in predose hypoxanthine between good and poor responders, but this was not commented on. Because of the involvement of purine metabolites in differential behaviour between good responders and poor responders, a pharmacogenomic study was initiated looking for SNPs in nine genes associated with purine biosynthesis, degradation or transport. A total of 51 SNPs were found in the adenosine kinase gene that were associated with differences in platelet aggregation during aspirin therapy, with the most strongly 
associated SNP being the intronic variant rs16931294. The less common $\mathrm{G}$ allele was associated with higher platelet aggregation, i.e. poor response, than the more common $\mathrm{A}$ allele. Exploratory studies in 74 participants showed that the $\mathrm{G}$ allele of rs16931294 was significantly associated with higher pre-dose levels of AMP, xanthine and hypoxanthine than the A allele. The less common $G$ allele was also significantly associated with higher post-aspirin levels of inosine and guanosine. The latter result is perplexing as, although the inosine data correlates with the earlier finding of higher post-dose inosine levels in poor responders, inosine is reported to inhibit platelet aggregation, and therefore further work was recommended.[56] However, this still represents an interesting example of the pharmacometabonomics-informed pharmacogenomics paradigm. Somewhat surprisingly, a subset of the authors published a review focused on just this paper in the very next edition of the same journal.[48]

A third paper[49] reported on the use of LC-MS to profile primary and secondary amine metabolites in serum samples from healthy volunteers in the same HAPI cohort, preand post-aspirin dosing: 80 volunteers were in a discovery cohort and 125 volunteers in a follow-up, replication cohort. In the discovery cohort, pre-dose serum levels of taurine and serotonin were significantly correlated with higher post-aspirin, collagen-induced, platelet aggregation. In the replication cohort, pre-dose serum levels of serotonin, but not taurine, were significantly correlated with higher post-aspirin, collagen-induced, platelet aggregation. A further study was conducted in a cohort of 38 healthy subjects from the general, non-Amish population. Whilst there was no difference in platelet aggregation in platelet rich plasma (PRP) between subjects with the highest or lowest PRP serotonin levels pre-aspirin, inhibition of collagen-stimulated platelet aggregation was significantly lower in individuals with higher pre-aspirin levels of serotonin, after ex vivo incubation of the PRP with aspirin (53 $\mu \mathrm{mol} / \mathrm{l}$ for 10 minutes).

\section{Predictive Metabonomics}

Figure 3a gives a conceptual depiction of metabonomics, which is defined as "The study of the metabolic response of organisms to disease, environmental change or genetic modification'.[12] The figure depicts the alteration to the metabolite profiles of individuals as they undergo an intervention, which could be drug administration, a change in diet, the adoption of a new exercise regime etc. Figure $3 \mathrm{~b}$ shows the corresponding depiction of pharmacometabonomics, where the pre-dose metabolic profiles of individuals enable some degree of prediction of the post-dose response of those individuals to drug administration. It is worth emphasizing here that drug administration is just one example of a host of interventions, the outcome of which could theoretically be predicted using pre-intervention metabolite profiling. This general class of experiments is defined as predictive metabonomics. The definition of predictive metabonomics, by analogy to the definition of pharmacometabonomics is: 'the prediction of the outcome of an intervention in an individual based on a mathematical model of pre-intervention metabolite signatures'. This is thus a generalisation of the definition of pharmacometabonomics,[16] and it is clear that pharmacometabonomics is just one special case (where the intervention is drug dosing) of the more general class of experiments that we term predictive metabonomics. In predictive metabonomics the intervention could be change of diet, exercise, a change in environment or simply, the passage of time. 
An excellent example of predictive metabonomics is provided by the work of WangSattler et al,[53] who showed that low levels of glycine and lysophosphatidylcholine (LPC) (18:2) at baseline predicted the risks of developing impaired glucose tolerance (IGT) and / or type-2 diabetes (T2D) in hundreds of subject from the population-based Cooperative Health Research in the Region of Augsburg cohort (KORA) cohort. This is an example of predictive metabonomics where the intervention is simply the passage of time, and the prediction is of whether a person will, or will not, suffer the onset of disease within that time period. Remarkably, as much as seven years prior to disease onset, glycine and LPC were also shown to be predictors of glucose tolerance. Glycine, LPC and acetylcarnitine were also found to have significantly altered levels in IGT subjects. In a metabonomics-guided genomics study, this same study also showed that seven T2D-related genes were functionally associated with the three IGT discriminating molecules. The ability to predict disease onset or progression using baseline metabolic profiles is a very exciting prospect for preventive medicine in the future.

\section{Future Perspectives}

Pharmacometabonomics is less than ten years old but is already showing a significant amount of promise. Published examples of clinical pharmacometabonomics have demonstrated the prediction of drug PK, metabolism, efficacy and toxicity, prior to drug dosing. In addition, several studies have developed the exciting concept of pharmacometabonomics-informed pharmacogenomics. It is already clear that pharmacometabonomics should not be thought of as competitive to pharmacogenomics, but rather they should be used synergistically together. It is also clear that pharmacometabonomics is just one special case of a broader set of predictive metabonomics experiments, which hold out the prospect of improved prediction of disease onset and progression (amongst many other things), which will be of immense value for medicine, and particularly for personalised or precision medicine, in the future. A further evolving concept is that of longitudinal pharmacometabonomics, where metabolic profiling is undertaken throughout a 'patient journey', in order to be better able to predict patient outcomes.[57]

Finally, a host of exciting, recent studies have used genome-wide association studies (GWAS) to relate genetic variation with human blood, serum and urine metabolite profiles on a large scale.[58-61] The linkage of variation of metabolite profiles with genetic as opposed to environmental variation provides new opportunities to link metabolic profiles to disease onset and treatment and the prediction of drug effects, via previous GWAS associations with these factors. This will be an exciting future area of research. Just one example of the potential of this approach is provided by a GWASmetabonomics study of metabolic traits associated with SNPs found in 835 Caucasian participants on the CoLaus study.[62] A key finding was of an association between urinary fucose levels and the presence of SNP rs492602 in the FUT2 gene, which encodes a fucosyltransferase enzyme. The FUT2 gene has already been associated with Crohn's disease, and it was proposed that elevated urinary fucose could be a biomarker for the dysbiosis associated with Crohn's disease, especially as fucose is known to influence human microbiome composition.

It is clear that pharmacometabonomics has a long way to go to be fully understood and explored as a research tool, and even further for it to be clinically validated in any 
setting, but we look forward to clinical utility of this important new development in the future.

\section{Executive Summary.}

\section{metabonomics}

- the study of the metabolic response of organisms to disease, environmental change or genetic modification

- a powerful method of interrogating changes in human physiology at a systems biology level

- metabonomics reflects the actual physiological status of a person at the point of sampling and not a possible future state, as with genomics

- the metabolic profiles of people reflect the influence of both their genomes and their environment and in particular, their microbiome

\section{pharmacometabonomics}

- the prediction of the outcome (for example, efficacy or toxicity) of a drug or xenobiotic intervention in an individual based on a mathematical model of preintervention metabolite signatures

- the metabolic profiling equivalent of pharmacogenomics

- first discovered in animals in 2000 and in humans in 2003, using nuclear magnetic resonance (NMR) spectroscopy methods, with the first publications in 2006 and 2009 respectively, so a very new area compared with pharmacogenomics

- pharmacometabonomics has been demonstrated to be able to give some degree of prediction of human drug PK, metabolism, safety and efficacy

- all studies to date are in the basic biomedical research phase and it will be some time before pharmacometabonomics biomarkers are used in clinical practice

\section{methodologies}

- both metabonomics and pharmacometabonomics can be approached in an unbiased, untargeted fashion (no pre-selection of metabolites to measure), or in a targeted approach (pre-selection of metabolites to measure)

- powerful analytical technologies such as NMR spectroscopy, gas chromatography - mass spectrometry, or ultra performance liquid chromatography - mass spectrometry are used to measure the metabolite profiles in human and animal biofluids and tissues

- multivariate statistical methodologies such as principal components analysis or projection to latent structures are used to help to analyse the complex data sets that emerge from pharmacometabonomics experiments

- care must be taken to ensure that studies are sufficiently validated from a statistical perspective and that the false discovery rate is known and controlled

\section{future developments}

- the recent development of pharmacometabonomics-informed pharmacogenomics is an exciting one and has shown the synergy that exists between these two key platforms for personalised medicine

- pharmacometabonomics is expected to deliver more insights into the complex interplay between the human genome and microbiome and the influence of both factors on diseases and their response to drug treatment

- pharmacometabonomics is just one specific example of a broader class of 
experiments termed predictive metabonomics, where pre-event metabolite profiles may be used to predict post-event outcomes; in the special case of pharmacometabonomics, the event is drug dosing

- exciting examples of predictive metabonomics are emerging, for instance, the ability to predict the onset of diseases such as Type 2 Diabetes years in advance

\section{Financial and Competing Interests.}

Jeremy Everett is a co-inventor on a recently granted patent on pharmacometabonomics (EP1540560). No writing assistance was utilized in the production of this manuscript.

\section{Acknowledgements}

I would like to acknowledge a long and fruitful collaboration with Professor Jeremy Nicholson at Imperial College, and also with Professor Elaine Holmes, Professor John Lindon and Professor Ian Wilson in the Department of Surgery and Cancer at Imperial. I would also like to acknowledge the help and support of the other members of the Medway Metabonomics Research Group, especially the co-leaders, Professor Frank Pullen and Dr Ruey Leng Loo. Finally, I would like to thank Dr Stefano Balducci and Ms Dorsa Varshavi for their contributions to our work on metabolic profiling of diabetic patients.

\section{Glossary of Abbreviations and Acronyms}

\begin{tabular}{|l|l|}
\hline term & meaning \\
\hline 1D & one-dimensional \\
\hline 1H & hydrogen-1 \\
\hline 2D & two-dimensional \\
\hline AMP & adenosine monophosphate \\
\hline COSY & COrrelated SpectroscopY \\
\hline CYP & cytochrome P450 \\
\hline EC & electrochemical analysis \\
\hline GC & gas chromatography \\
\hline GWAS & genome-wide association studies \\
\hline HPLC & high performance liquid chromatography \\
\hline JRES & J-resolved \\
\hline LC & liquid chromatography \\
\hline MS & mass spectrometry \\
\hline NMR & nuclear magnetic resonance spectroscopy \\
\hline PK & pharmacokinetics \\
\hline SNP & single nucleotide polymorphism \\
\hline T2D or T2DM & type 2 diabetes mellitus \\
\hline TOF & time-of-flight \\
\hline UPLC & ultra performance liquid chromatography \\
\hline
\end{tabular}




\section{References}

1. Jorgensen JT. A challenging drug development process in the era of personalized medicine. Drug Discovery Today, 16(19-20), 891-897 (2011).

2. Lazarou J, Pomeranz BH, Corey PN. Incidence of adverse drug reactions in hospitalized patients - A meta-analysis of prospective studies. Jama-Journal of the American Medical Association, 279(15), 1200-1205 (1998).

3. Miguel A, Azevedo LF, Araujo M, Pereira AC. Frequency of adverse drug reactions in hospitalized patients: a systematic review and meta-analysis. Pharmacoepidemiology and Drug Safety, 21(11), 1139-1154 (2012).

4. Sultana J, Cutroneo P, Trifiro G. Clinical and economic burden of adverse drug reactions. Journal of pharmacology \& pharmacotherapeutics, 4(Suppl 1), S73-77 (2013).

5. Lee JW, Aminkeng F, Bhavsar AP et al. The emerging era of pharmacogenomics: current successes, future potential, and challenges. Clinical Genetics, 86(1), 21-28 (2014).

6. Pirmohamed M. Personalized pharmacogenomics: predicting efficacy and adverse drug reactions. Annual review of genomics and human genetics, 15, 349370 (2014).

7. Pokorska-Bocci A, Stewart A, Sagoo GS, Hall A, Kroese M, Burton H. 'Personalized medicine': what's in a name? Personalized Medicine, 11(2), 197-210 (2014).

8. Joseph PG, Pare G, Ross S, Roberts R, Anand SS. Pharmacogenetics in Cardiovascular Disease: The Challenge of Moving From Promise to Realization Concepts Discussed at the Canadian Network and Centre for Trials Internationally Network Conference (CANNeCTIN), June 2009. Clinical Cardiology, 37(1), 48-56 (2014).

9. $\quad$ Maruthur NM, Gribble MO, Bennett WL et al. The Pharmacogenetics of Type 2 Diabetes: A Systematic Review. Diabetes Care, 37(3), 876-886 (2014).

10. Perlis RH. Pharmacogenomic Testing and Personalized Treatment of Depression. Clinical Chemistry, 60(1), 53-59 (2014).

11. Carr DF, Alfirevic A, Pirmohamed M. Pharmacogenomics: Current State-of-theArt. Genes, 5(2), 430-443 (2014).

12. Lindon J, Nicholson J, Holmes E, Everett J. Metabonomics: Metabolic processes studied by NMR spectroscopy of biofluids. Concepts in Magnetic Resonance, 12(5), 289-320 (2000).

13. Lindon J, Nicholson J, Everett J. NMR spectroscopy of biofluids. In: Annual Reports on Nmr Spectroscopy, Vol 38. (1999) 1-88.

14. Fiehn O. Metabolomics--the link between genotypes and phenotypes. Plant Mol Biol, 48(1-2), 155-171 (2002).

15. Lindon JC, Nicholson JK, Holmes E. The handbook of metabonomics and metabolomics (Elsevier, Amsterdam ; Oxford, 2007).

16. Clayton $\mathrm{T}$, Lindon J, Cloarec $\mathrm{O}$ et al. Pharmaco-metabonomic phenotyping and personalized drug treatment. Nature, 440(7087), 1073-1077 (2006).

17. Kaddurah-Daouk R, Boyle SH, Matson W et al. Pretreatment metabotype as a predictor of response to sertraline or placebo in depressed outpatients: a proof of concept. Transl Psychiatry, 1, 1-7 (2011).

18. Kaddurah-Daouk R, Baillie RA, Zhu H et al. Enteric microbiome metabolites correlate with response to simvastatin treatment. PLoS One, 6(10), e25482 (2011). 
19. Wishart DS. Characterization of biopharmaceuticals by NMR spectroscopy. TracTrends in Analytical Chemistry, 48, 96-111 (2013).

20. Sumner LW, Amberg A, Barrett D et al. Proposed minimum reporting standards for chemical analysis. Metabolomics, 3(3), 211-221 (2007).

21. Dunn WB, Erban A, Weber RJM et al. Mass appeal: metabolite identification in mass spectrometry-focused untargeted metabolomics. Metabolomics, 9(1), S44S66 (2013).

22. Creek DJ, Dunn WB, Fiehn 0 et al. Metabolite identification: are you sure? And how do your peers gauge your confidence? Metabolomics, 10(3), 350-353 (2014).

23. Everett JR. A New Paradigm for Known Metabolite Identification in Metabonomics/Metabolomics: Metabolite Identification Efficiency. (Ed.^(Eds) (Computational and Structural Biotechnology Journal, 2015)

24. Burton K, Everett J, Newman M, Pullen F, Richards D, Swanson A. On-line liquid chromatography coupled with high field NMR and mass spectrometry (LC-NMRMS): a new technique for drug metabolite structure elucidation. Journal of Pharmaceutical and Biomedical Analysis, 15(12), 1903-1912 (1997).

25. Everett JR, Balduccci S, Varshavi D. collaboration work. (Ed.^(Eds) (Unpublished collaboration study, 2014)

26. Claridge T. High-Resolution NMR Techniques in Organic Chemistry (Elsevier, Oxford, UK, 2009).

27. Wishart DS, Jewison T, Guo AC et al. HMDB 3.0-The Human Metabolome Database in 2013. Nucleic Acids Research, 41(D1), D801-D807 (2013).

28. Craig A, Cloarec O, Holmes E, Nicholson JK, Lindon JC. Scaling and normalization effects in NMR spectroscopic metabonomic data sets. Anal Chem, 78(7), 22622267 (2006).

29. Dona AC, Jimenez B, Schaefer H et al. Precision High-Throughput Proton NMR Spectroscopy of Human Urine, Serum, and Plasma for Large-Scale Metabolic Phenotyping. Analytical Chemistry, 86(19), 9887-9894 (2014).

30. Emwas A-HM, Salek RM, Griffin JL, Merzaban J. NMR-based metabolomics in human disease diagnosis: applications, limitations, and recommendations. Metabolomics, 9(5), 1048-1072 (2013).

31. Lindon JC, Nicholson JK. Spectroscopic and Statistical Techniques for Information Recovery in Metabonomics and Metabolomics. Annual Review of Analytical Chemistry, 1, 45-69 (2008).

32. Clayton TA, Baker D, Lindon JC, Everett JR, Nicholson JK. Pharmacometabonomic identification of a significant host-microbiome metabolic interaction affecting human drug metabolism. Proceedings of the National Academy of Sciences of the United States of America, 106(34), 14728-14733 (2009).

33. Wilson ID. Drugs, bugs, and personalized medicine: pharmacometabonomics enters the ring. In: Proc Natl Acad Sci U S A. (United States, 2009) 14187-14188.

34. Everett JR, Loo RL, Pullen FS. Pharmacometabonomics and personalized medicine. Ann Clin Biochem, 50, 523-545 (2013).

35. Nicholson JK, Wilson ID, Lindon JC. Pharmacometabonomics as an effector for personalized medicine. Pharmacogenomics, 12(1), 103-111 (2011).

36. Fanos V, Barberini L, Antonucci R, Atzori L. Pharma-metabolomics in Neonatology: is it a Dream or a Fact? Current Pharmaceutical Design, 18(21), 2996-3006 (2012). 
37. Antonucci R, Pilloni MD, Atzori L, Fanos V. Pharmaceutical research and metabolomics in the newborn. Journal of Maternal-Fetal \& Neonatal Medicine, 25, 22-26 (2012).

38. Phapale PB, Kim SD, Lee HW et al. An integrative approach for identifying a metabolic phenotype predictive of individualized pharmacokinetics of tacrolimus. Clin Pharmacol Ther, 87(4), 426-436 (2010).

39. Rahmioglu N, Le Gall G, Heaton J et al. Prediction of Variability in CYP3A4 Induction Using a Combined H-1 NMR Metabonomics and Targeted UPLC-MS Approach. Journal of Proteome Research, 10(6), 2807-2816 (2011).

40. Shin KH, Choi MH, Lim KS, Yu KS, Jang IJ, Cho JY. Evaluation of Endogenous Metabolic Markers of Hepatic CYP3A Activity Using Metabolic Profiling and Midazolam Clearance. Clinical Pharmacology \& Therapeutics, 94(5), 601-609 (2013).

41. Kaddurah-Daouk R, Baillie RA, Zhu HJ et al. Lipidomic analysis of variation in response to simvastatin in the Cholesterol and Pharmacogenetics Study. Metabolomics, 6(2), 191-201 (2010).

42. Trupp M, Zhu H, Wikoff WR et al. Metabolomics reveals amino acids contribute to variation in response to simvastatin treatment. PLoS One, 7(7), e38386 (2012).

43. Ji Y, Hebbring S, Zhu H et al. Glycine and a glycine dehydrogenase (GLDC) SNP as citalopram/escitalopram response biomarkers in depression: pharmacometabolomics-informed pharmacogenomics. Clin Pharmacol Ther, 89(1), 97-104 (2011).

44. Abo R, Hebbring S, Ji Y et al. Merging pharmacometabolomics with pharmacogenomics using '1000 Genomes' single-nucleotide polymorphism imputation: selective serotonin reuptake inhibitor response pharmacogenomics. Pharmacogenet Genomics, 22(4), 247-253 (2012).

45. Kaddurah-Daouk R, Bogdanov MB, Wikoff WR et al. Pharmacometabolomic mapping of early biochemical changes induced by sertraline and placebo. Transl Psychiatry, 3, e223 (2013).

46. Zhu H, Bogdanov MB, Boyle SH et al. Pharmacometabolomics of response to sertraline and to placebo in major depressive disorder - possible role for methoxyindole pathway. PLoS One, 8(7), e68283 (2013).

47. Condray R, Dougherty GG, Keshavan MS et al. 3-Hydroxykynurenine and clinical symptoms in first-episode neuroleptic-naive patients with schizophrenia. International Journal of Neuropsychopharmacology, 14(6), 756-767 (2011).

48. Lewis JP, Yerges-Armstrong LM, Ellero-Simatos S, Georgiades A, KaddurahDaouk R, Hankemeier T. Integration of Pharmacometabolomic and Pharmacogenomic Approaches Reveals Novel Insights Into Antiplatelet Therapy. Clinical Pharmacology \& Therapeutics, 94(5), 570-573 (2013).

49. Ellero-Simatos S, Lewis JP, Georgiades A et al. Pharmacometabolomics reveals that serotonin is implicated in aspirin response variability. CPT: pharmacometrics \& systems pharmacology, 3, e125-e125 (2014).

50. Keun HC, Sidhu J, Pchejetski D et al. Serum Molecular Signatures of Weight Change during Early Breast Cancer Chemotherapy. Clinical Cancer Research, 15(21), 6716-6723 (2009).

51. Backshall A, Sharma R, Clarke SJ, Keun HC. Pharmacometabonomic profiling as a predictor of toxicity in patients with inoperable colorectal cancer treated with capecitabine. Clin Cancer Res, 17(9), 3019-3028 (2011). 
52. Winnike JH, Li Z, Wright FA, Macdonald JM, O'Connell TM, Watkins PB. Use of pharmaco-metabonomics for early prediction of acetaminophen-induced hepatotoxicity in humans. Clin Pharmacol Ther, 88(1), 45-51 (2010).

53. Wang-Sattler R, Yu Z, Herder C et al. Novel biomarkers for pre-diabetes identified by metabolomics. Molecular Systems Biology, 8 (2012).

54. Kema IP, de Vries EGE, Muskiet FAJ. Clinical chemistry of serotonin and metabolites. Journal of Chromatography B, 747(1-2), 33-48 (2000).

55. Wikoff WR, Frye RF, Zhu H et al. Pharmacometabolomics reveals racial differences in response to atenolol treatment. PloS one, 8(3), e57639-e57639 (2013).

56. Yerges-Armstrong LM, Ellero-Simatos S, Georgiades A et al. Purine Pathway Implicated in Mechanism of Resistance to Aspirin Therapy:

Pharmacometabolomics-Informed Pharmacogenomics. Clinical Pharmacology \& Therapeutics, 94(4), 525-532 (2013).

57. Nicholson JK, Everett JR, Lindon JC. Longitudinal pharmacometabonomics for predicting patient responses to therapy: drug metabolism, toxicity and efficacy. Expert Opin Drug Metab Toxicol, 8(2), 135-139 (2012).

58. Suhre K, Shin S-Y, Petersen A-K et al. Human metabolic individuality in biomedical and pharmaceutical research. Nature, 477(7362), 54-U60 (2011).

59. Suhre K, Wallaschofski H, Raffler J et al. A genome-wide association study of metabolic traits in human urine. Nature Genetics, 43(6), 565-U597 (2011).

60. Kettunen J, Tukiainen T, Sarin A-P et al. Genome-wide association study identifies multiple loci influencing human serum metabolite levels. Nature Genetics, 44(3), 269-U265 (2012).

61. Shin S-Y, Fauman EB, Petersen A-K et al. An atlas of genetic influences on human blood metabolites. Nature Genetics, 46(6), 543-550 (2014).

62. Rueedi R, Ledda M, Nicholls AW et al. Genome-wide association study of metabolic traits reveals novel gene-metabolite-disease links. PLoS genetics, 10(2), e1004132-e1004132 (2014). 